\title{
Logic-Based Interpretation of
}

\section{Context:}

\section{Modelling and Applications}

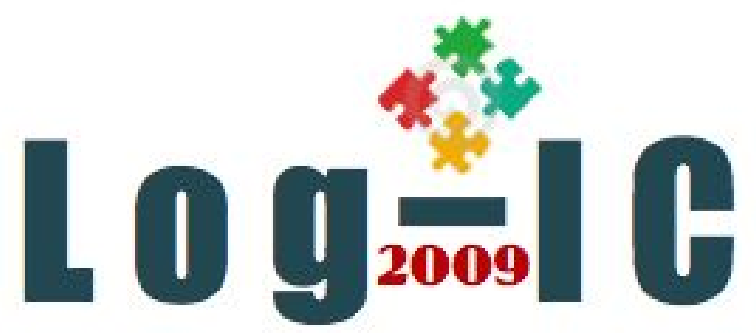

First International Workshop

14 September 2009, Potsdam, Germany

Alessandra Mileo

James P. Delgrande (Eds.) 



\section{Preface}

Context interpretation and context-based reasoning are key factors in the development of intelligent autonomous systems in a variety of applications. The ability to represent contextual factors, interpret them and combine them with other sources of knowledge are some of the challenges to enable intelligent systems achieve correct behavior. Much work has been done in application areas that make use of contextual information, such as pervasive computing, logic-based sensor fusion and data integration. As well, the theoretical foundations for context-based reasoning have been studied. However, there is still a great deal to do in context modeling, since generic context models for contextaware application development need to be further explored, as does the role of context reasoning with more recently emerging areas such as ontologies, including the semantic web, and approaches to belief change.

Context-dependent data can arise from different sources; for example it may be gathered by sensors or collected from different knowledge- or databases. The incompleteness and heterogenous nature of such data and the need for state-based context interpretation in dynamic systems suggest that nonmonotonic reasoning techniques can be a powerful tool for effective context-dependent reasoning. Given the increasing interest in hybrid knowledge representation formalisms as basis of the Semantic Web, it is interesting to consider proposals that assume hybrid formalisms combining Description Logics and Logic Programming as the basic representation framework for reasoning with (distributed) contexts.

The Log-IC'09 workshop provides a forum for researchers investigating contextaware applications and context-based reasoning with the goal of sharing and comparing their views on the efficacy of different context representation and context interpretation frameworks. Log-IC 2009 will also propose targeted discussions on the topic. Holding the workshop in conjunction with LPNMR 2009 (organized in Potsdam) has the additional advantage of reaching out to the logic programming community, facilitating collaboration between different formalisms for context-based reasoning.

Apart from the regular and short paper presentations, the workshop also welcomes Gerhard Brewka (University of Leipzig, Germany) and Grigoris Antoniou (University of Crete, Greece) as invited speakers. We plan a panel to initiate discussions on dealing with contexts both in theoretical and application-oriented perspectives.

Within these proceedings you can find the five papers that were accepted for publication by our programme committee and the abstract of our invited talk.

The programme committee and organisers wish to thank all the authors who submitted papers, the invited speaker, the panel members, the reviewers, all participants and everyone who contributed to the success of this workshop.

September 2009

Alessandra Mileo

James P. Delgrande

Organisers

Log-IC'09 


\title{
Organisation
}

\section{Executive Committee}

Workshop Chairs:

\author{
Alessandra Mileo \\ (Universitá degli Studi di Milano-Bicocca, Milan, Italy) \\ James P. Delgrande \\ (Simon Fraser University, Burnaby, Canada)
}

\section{Programme Committee}

Marcello Balduccini

Leopoldo Bertossi

Gerhard Brewka

Pedro Cabalar Fernandez

Amedeo Cesta

Marina de Vos

James P. Delgrande

Wolfgang Faber

Jorge Lobo

Robert Mercer

Tommie Meyer

Alessandra Mileo

Torsten Schaub

Kewen Wang

Nic Wilson

Stefan Woltran
Kodak Research Labs, Rochester, NY, USA

Carleton University, Canada

University of Leipzig, Germany

Corunna University, Galicia, Spain

ISTC-CNR, Rome, Italy

University of Bath, UK

Simon Fraser University, Vancouver, Canada

Department of Mathematics, University of Calabria

IBM T. J. Watson Research Center, Hawthorne, NY, USA

University of Western Ontario, Canada

Meraka Institute, Pretoria, South Africa

University of Milan-Bicocca, Italy

University of Potsdam, Germany

Griffith University, Brisbane, Australia

University College, Cork, Ireland

Vienna University of Technology, Austria 


\section{Table of Contents}

\section{Invited Speaker}

Nonmonotonic Multi-Context Systems: State of the Art and Future Challenges . . 3 Gerhard Brewka (University of Leipzig, D)

Reasoning about Context in Ambient Intelligence Environments . . . . . . . . 4 Grigoris Antoniou (University of Crete, GR)

\section{Research Papers}

From Symbolic to Probabilistic Models . . . . . . . . . . . . . . . . .

S. Bader (Rostock University, Germany) and C. Burghardt (Rostock

University, Germany) and T. Kirste (Rostock University, Germany)

Towards Diagnosing Inconsistency in Nonmonotonic Multi-Context Systems . . .

T. Eiter (Technisce Universität Wien, Austria) and M. Fink (Technisce Universität Wien, Austria), P. Schüller (Technisce Universität Wien, Austria) and A. Weinzierl (Technisce Universität Wien, Austria)

Relations as Context to Improve Multi-Target Tracking and Activity Recognition

C. Manfredotti (University of Toronto, Canada) and E. Messina (University of Milano-Bicocca, Italy) and D. Fleet (University of Toronto, Canada)

Who, Where, What, and When: Towards Characterizing Contexts in Pervasive

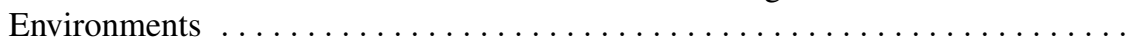

A. Mileo (University of Milano-Bicocca

A Constuctive Modal Semantics for Contextual Verification . . . . . . . . . . . . 
VIII

ISSN 1613-0073 (C) 2009 for the individual papers by the papers' authors. 


\section{Part I}

\section{Invited Speaker}





\title{
Nonmonotonic Multi-Context Systems: State of the Art and Future Challenges
}

\author{
Gerhard Brewka \\ Intelligent Systems Department \\ Computer Science Institute \\ University of Leipzig, Germany \\ brewka@informatik.uni-leipzig.de
}

\section{Invited Abstract}

In our talk we will describe recent developments in the area of multi-context systems (MCS). We will first discuss nonmonotonic generalizations of multi-context systems as introduced by Brewka and Eiter. These systems allow us to combine a large class of monotonic and nonmonotonic knowledge representation formalisms in a principled and modular way. The information flow among the component formalisms is modelled using nonmonotonic bridge rules which generalize the rules originally introduced by Giunchiglia and colleagues.

We then present a more recent approach, argumentation context systems (ACS), which focus on a particular form of component formalisms, namely Dung argumentation frameworks. In this respect ACS are more restricted than nonmonotonic MCS. However, ACS go beyond MCS in at least two important aspects: they include a variety of inconsistency handling methods integrating possibly conflicting information from different components, and they allow for more drastic effects of parent modules on child modules, including the deletion of arguments and attacks and the specification of reasoning mode and semantics. Technically this is achieved by introducing a new type of components called mediators and a context-dependent form of abstract argumentation.

The natural next step is to combine these ideas, that is to generalize both nonmonotonic MCS and ACS to a new form of mediator based MCS. We report on our current ideas about how this can be done. 


\title{
Reasoning about Context in Ambient Intelligence Environments
}

\author{
Grigoris Antoniou \\ Institute of Computer Science (FORTH) \\ University of Crete, Greece \\ antonioudics. forth.gr
}

\section{Invited Abstract}

The imperfect nature of context in Ambient Intelligence (AmI) environments, and the special characteristics of the entities that possess and share the available context information render contextual reasoning a very challenging task. The accomplishment of this task requires formal models that handle the involved entities as autonomous logicbased agents, and provide methods for handling the imperfect and distributed nature of context.

In this talk, we (a) review the reasoning challegenes associated with these environments; (b) propose a solution on reasoning about context based on the Multi-Context Systems formalism and defeasible reasoning, and (c) report on a prototypical implementation on small devices. 


\section{Part II}

\section{Research Papers}





\title{
From Symbolic to Probabilistic Models
}

\author{
Sebastian Bader, Christoph Burghardt, and Thomas Kirste \\ MMIS, Rostock University, Germany \\ first.lastname@uni-rostock.de, http://mmis.informatik.uni-rostock.de
}

\begin{abstract}
We argue, that generative probabilistic models should be used to detect user activities, and we discuss two approaches to create those model from symbolic descriptions.
\end{abstract}

\section{Motivation}

In many application areas, a computer needs to recognise the user's current activity. Examples are the automatic creation of diaries, user assistance in instrumented environments and many others. Unfortunately, activity recognition is by no means a simple problem, because we have to deal with the problems of noisy sensor data, incomplete descriptions of the domain, unpredictable behaviours of humans and many others. In this paper, we argue that we need (i) generative probabilistic models for activity recognition and (ii) high-level description of these models in a human readable form. And we show two possible approaches currently under investigation in our lab.

\section{Probabilistic Models and Abstract Description}

As mentioned above, we have to deal with noisy sensor data while trying to recognise the user's activity. Probabilistic models, for example hidden Markov models or more general dynamic Bayesian networks, have been applied successfully. We believe that generative models should be used for the high-level activity recognition because they allow an easy integration of prior knowledge and we can not only recognise the user's activity but also predict and simulate it.

Unfortunately, probabilistic models quickly become rather complex. Therefore, they are neither easy to construct nor to debug by humans. Both problems could be solved, if we were able to automatically construct complex models based on a symbolic description, and to extract such a description from a (trained) model later.

Below we discuss the creation of hidden Markov models (HMM) from (a) grammars and (b) STRIPS descriptions [2]. While the first is a top-down approach (starting at the highest level, which is then detailed) the latter works in a bottom-up fashion (starting from atomic actions that are composed into sequences).

We propose to use a extension of probabilistic context free grammars [2] (EPCFG) to define the language of human activities of daily living such that 


$$
\begin{gathered}
\text { Day } \stackrel{0.7}{\rightarrow} \text { WDay } \mid \stackrel{0.3}{\rightarrow} \text { Weekend } \\
\text { WDay } \stackrel{0.3}{\rightarrow} \text { Car, work }[8 h], \text { Car } \\
\text { Car } \stackrel{0.5}{\rightarrow} \text { carfast }[1 \mathrm{~min}], @ 0.9 \\
\text { Car } \stackrel{0.3}{\rightarrow} \text { carslow }[1 \min ], @ 0.9 \\
\text { Car } \stackrel{0.2}{\rightarrow} \text { carstop }[1 \min ], @ 0.9
\end{gathered}
$$

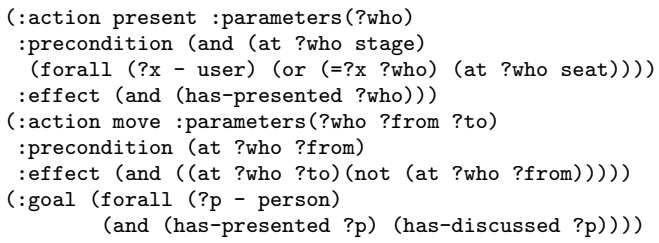

Fig. 1. Excerpts of an extended grammar describing a human's life (on the left) and a STRIPS formalisation of a smart meeting room (on the right).

the underlying terminal symbols correspond to observable primitive activities. I.e., for each of them we can define a probability distribution over the raw sensor data. Figure 1 shows a grammar which is annotated by probabilities and timing information. For a speedometer, the terminal carfast can be defined as a normal distribution with mean "50 km/h" and variance of " $10 \mathrm{~km} / \mathrm{h}$ ". The EPCFG is translated into a hierarchical HMM, which then is flattened. The resulting HMM can be used for annotation. But, we can also assign labels to the states of the model which allows the detection of high-level activities.

As a second approach, we propose to use STRIPS operators as known from planning $[2,1]$. These operators define pre- and post-conditions of actions. A simple example is shown in Figure 1 on the right, in which we model the activities during meetings. Those meetings are hard to model using grammars, because all possible sequences of actions have to be modelled. By employing the STRIPS formalism, we can generate all possible meeting sequences via expansion from an initial state, given the number of participants and the agenda of the meeting. This allows a straight-forward integration of prior knowledge of the domain, e.g., social norms. Those sequences of states can easily be modelled using HMMs.

\section{Discussion}

We have discussed two approaches to create probabilistic models for high-level activity recognition based on symbolic descriptions. Both allow the automatic generation of such models based on a symbolic description. The training and the extraction of symbolic descriptions from the revised models needs to be investigated in the future. Furthermore, we need to evaluate our approach using real problems.

Acknowledgements We like to thank two anonymous reviewers for their comments on an earlier version of this paper.

\section{References}

1. Ch. Burghardt and Th. Kirste: Inferring intentions in generic context-aware systems. Proc. of MUM'07, 50-54, Oulu, Finland (2007)

2. S. Russell and P. Norvig: AI: A Modern Approach. Prentice Hall (2003)

ISSN 1613-0073 (C) 2009 for the individual papers by the papers' authors. 


\title{
Towards Diagnosing Inconsistency in Nonmonotonic Multi-Context Systems ${ }^{\star}$
}

\author{
Thomas Eiter, Michael Fink, Peter Schüller, and Antonius Weinzierl \\ Institute of Information Systems \\ Technische Universität Wien \\ Favoritenstrasse 11, A-1040 Vienna, Austria \\ \{eiter,fink, schueller, weinzierl\}@kr.tuwien.ac.at
}

\begin{abstract}
In multi-context systems, heterogeneous contexts interact via nonmonotonic bridge rules. We seek to understand and give reasons for inconsistencies in such systems by means of diagnosis. For this purpose, we propose notions of consistency-based and abduction-based diagnosis, where diagnoses are characterized by sets of bridge rules. Interestingly, the notions of consistency-based and abduction-based diagnoses lead to the same sets of bridge rules which are potentially erroneous.
\end{abstract}

Introduction. Multi-context systems (MCSs) as defined by Brewka and Eiter[1] are a powerful framework for integrating heterogeneous nonmonotonic logics like ontologies, databases or answer set programs. MCSs can represent intercontextual information flow and express reasoning with respect to contextual information. The formalism allows for decentralized systems which use pointwise information exchange and consist of multiple components like, for instance, business logics, agents, or knowledge bases in general.

An MCS consists of contexts and bridge rules. Each context is a knowledge base with an underlying (nonmonotonic) logic providing its semantics in terms of belief sets. Contexts interact through nonmonotonic bridge rules of the form

$$
\left(c_{s}: s\right) \leftarrow\left(c_{1}: p_{1}\right), \ldots,\left(c_{j}: p_{j}\right), \operatorname{not}\left(c_{j+1}: p_{j+1}\right), \ldots, \operatorname{not}\left(c_{m}: p_{m}\right) .
$$

where $c_{s}, c_{1} \ldots, c_{m}$ are names of contexts and $s, p_{1}, \ldots, p_{m}$ are beliefs of the respective contexts. Intuitively, rule (1) is applicable wrt. belief sets $S_{1}, \ldots, S_{m}$ of the respective contexts, if $p_{i} \in S_{i}$, for $1 \leq i \leq j$, and $p_{k} \notin S_{k}$, for $j+1 \leq k \leq m$. If the rule is applicable, then its head $s$ is added to the knowledge base of $c_{s}$.

Example 1. Assume a health care decision support system which contains the following contexts: a patient history database $C_{1}$, a blood and X-Ray analysis database $C_{2}$, a description logic ontology of diseases $C_{3}$, and a disjunctive logic program implementing an expert system $C_{4}$ which suggests proper treatments.

* This work was supported by the Vienna Science and Technology Fund (WWTF) under grant ICT 08-020. 
Consider the following knowledge bases for these contexts, focusing on pneumonia (a lung disease treatable by antibiotics):

$$
\begin{aligned}
C_{1}= & \{\text { allergy_strong_antibiotic }\} \\
C_{2}= & \left\{\neg \text { blood_marker, } x r a y \_p n e u m o n i a\right\} \\
C_{3}= & \{\text { atypical_pneumonia } 5 \text { pneumonia } \sqcap \text { marker }\} \\
C_{4}= & \{\text { give_s } \vee \text { give_w } \leftarrow \text { need_antibiotic. } \\
& \text { give_s } \leftarrow \text { need_strong. } \\
& \perp \leftarrow \text { give_s, not allow_strong. } \\
& \text { nothing_required } \leftarrow \text { not need_antibiotic, not need_strong. }\}
\end{aligned}
$$

Contexts $C_{1}$ and $C_{2}$ provide the information that the patient is allergic to strong antibiotics, that a certain blood marker is not present, and that pneumonia was detected in the X-Ray. $C_{3}$ classifies atypical pneumonia as a combination of pneumonia and the presence of a blood marker. $C_{4}$ suggests a medication which is either a strong antibiotic $s$, a weak antibiotic $w$, or no medication at all.

The bridge rules of the MCS are given as:

$$
\begin{aligned}
& r_{1}=(3: \text { pneumonia }(p)) \leftarrow(2: \text { xray_pneumonia }) . \\
& r_{2}=(3: \text { marker }(p)) \quad \leftarrow(2: \text { blood_marker }) . \\
& r_{3}=(4: \text { need_antibiotic }) \leftarrow(3: \text { pneumonia }(p)) . \\
& r_{4}=(4: \text { need_strong }) \quad \leftarrow(3: \text { atypical_pneumonia }(p)) . \\
& r_{5}=(4: \text { allow_strong }) \quad \leftarrow \operatorname{not}(1: \text { allergy_strong_antibiotic }) .
\end{aligned}
$$

Rules $r_{1}$ and $r_{2}$ provide input for disease classification to the ontology, $r_{3}$ and $r_{4}$ link disease information with medication requirements, and $r_{5}$ relates acceptance of strong antibiotics with an allergy check on the patient database.

The semantics of MCSs is defined in terms of equilibria. An equilibrium consists of a belief state, i.e., a belief set $S_{i}$ for each context $C_{i}$, which is acceptable for $C_{i}$ under the addition of beliefs from applicable bridge rules.

Example 2. In our example there exists exactly one equilibrium $S$, and rules $r_{1}$ and $r_{3}$ are applicable wrt. $S$ (for $C_{3}$, a belief state $S_{3}$ consists of all class instances):

$$
\begin{aligned}
S= & \left(\{\text { allergy_strong_antibiotic }\},\left\{\neg \text { blood_marker }, x r a y \_p n e u m o n i a\right.\right. \\
& \{\text { pneumonia }(p)\},\{\text { need_antibiotic }, \text { give_w }\}) .
\end{aligned}
$$

Inconsistency in an MCS is the lack of an equilibrium. As the interaction and combination of heterogeneous systems can easily lead to unforeseen and intricate effects, inconsistency is a major-according to our knowledge unaddressedproblem in MCSs. In order to provide support for restoring consistency, we seek to understand and give reasons for inconsistency by means of diagnosis.

Example 3. As a running example, we consider a slightly modified version of Example 1, where the blood serum analysis shows presence of the blood marker:

$$
C_{2}=\{\text { blood_marker, xray_pneumonia }\} .
$$

This MCS is inconsistent since $r_{2}$ and $r_{4}$ become applicable, which require that strong antibiotic $s$ is applied. This is in conflict with the patient's allergy. 
We assume that every context is consistent without the influence of bridge rules, therefore we characterize reasons for inconsistency in terms of bridge rules.

Definition 1. Let $M$ be an MCS and $R$ a set of bridge rules. We write $R=_{M} \perp$ to say that the semantics of $M$ using $R$ as its set of bridge rules yields inconsistency. We write $R \not \forall_{M} \perp$ to say that $M$ using $R$ as its set of bridge rules has an equilibrium. In the following, $B R$ denotes the original set of bridge rules of $M$, and heads $(R)$ denotes the set of heads of rules in $R$ transformed to facts.

Diagnoses and Explanations for Inconsistency. In nonmonotonic reasoning, forcing rules to be applicable (or forcing them to be not applicable) can cause and prevent inconsistency. For our consistency-based diagnosis, we therefore consider pairs of sets of bridge rules, s.t. deactivating the rules in the one set and forcing the rules in the other to be active allows to establish consistency (i.e. an equilibrium) in the system.

Definition 2. A diagnosis $D^{ \pm}$of an $M C S M$ wrt. $B R$ is a pair $D^{ \pm}=\left(R^{-}, R^{+}\right)$, where $R^{-}, R^{+} \subseteq B R$ and $R^{-} \cap R^{+}=\emptyset$ s.t. $B R \backslash R^{-} \cup$ heads $\left(R^{+}\right) \not \nvdash_{M} \perp$.

Noticeably, this definition resembles the notion of anti-explanation [2], as well as the answer set program debugging approach in [3].

Example 4. Notable diagnoses in our running example are the following:

$$
\left(\left\{r_{1}\right\}, \emptyset\right),\left(\left\{r_{2}\right\}, \emptyset\right),\left(\left\{r_{4}\right\}, \emptyset\right) \text {, and }\left(\emptyset,\left\{r_{5}\right\}\right) \text {. }
$$

Accordingly, deactivating $r_{1}, r_{2}$, or $r_{3}$, resp. forcing $r_{5}$ to be active, will result in a consistent MCS. All other diagnoses are pointwise supersets thereof.

A research issue are preferred diagnoses wrt. an application domain. Minimality criteria (e.g. applied to preference orderings) can be used for this purpose. For domains where the removal of bridge rules is preferred to the forced activation of unjustified rules, we specialize $D^{ \pm}$to obtain diagnoses of the form $\left(R^{-}, \emptyset\right)$ only. We compare such diagnoses using subset-minimality as preference criterion.

Definition 3. An s-diagnosis ${ }^{1} D^{-}$of an $M C S M$ wrt. $B R$ is any set $R^{-} \subseteq B R$ s.t. $B R \backslash R^{-} \not \models_{M} \perp$. An s-diagnosis is minimal iff it is minimal wrt. $\subseteq$.

Example 5. Minimal s-diagnoses in our example are $\left\{r_{1}\right\},\left\{r_{2}\right\}$ and $\left\{r_{4}\right\}$.

Motivated by abduction-based diagnosis, we consider an explanation as a pair of sets of rules whose joint (de-)activation reproduces the observed inconsistency.

Definition 4. An inconsistency explanation $E^{ \pm}$of an MCS $M$ wrt. BR is a tuple $E^{ \pm}=\left(R^{-}, R^{+}\right)$, where $R^{-}, R^{+} \subseteq B R$ and $R^{-} \cap R^{+}=\emptyset$ s.t. $R^{-} \cup$ heads $\left(R^{+}\right) \models{ }_{M} \perp$.

Again we specialize the definition to the first component. In this case we additionally require that an explanation has no consistent superset, to avoid reproducing irrelevant inconsistencies. For instance, the program $\{a \leftarrow$ not $a$.$\} is inconsistent$ under the answer set semantics, but its superset $\{a \leftarrow$ not $a . a$.$\} is consistent.$

\footnotetext{
${ }_{1}$ The prefix s stands for simple.
} 
Definition 5. An s-inconsistency explanation $E^{+}$of an MCS $M$ wrt. BR is any set $R^{-} \subseteq B R$ s.t. $R^{-} \models_{M} \perp$ and there exists no $R^{\prime}$ s.t. $R^{-} \subset R^{\prime} \subseteq B R$ and $R^{\prime} \not \forall_{M} \perp$. An s-inconsistency explanation is minimal iff it is minimal wrt. $\subseteq$.

Example 6. The only minimal s-inconsistency explanation $E^{+}$in our running example is $\left\{r_{1}, r_{2}, r_{4}\right\}$; its rules are thus necessary to derive inconsistency.

The union of all minimal diagnoses (explanations) is a set of rules relevant for repairing (causing) inconsistency. Interestingly, these unions coincide (cf. also our example with minimal diagnoses $\left\{r_{1}\right\},\left\{r_{2}\right\},\left\{r_{4}\right\}$, resp. explanation $\left.\left\{r_{1}, r_{2}, r_{4}\right\}\right)$.

Theorem 1. For an inconsistent MCS, the unions of all minimal s-diagnoses $D_{m}^{-}$ and all minimal s-inconsistency explanations $E_{m}^{+}$coincide, i.e., $\bigcup D_{m}^{-}=\bigcup E_{m}^{+}$.

Discussion and Future Work. Minimal $D^{-}$diagnoses, i.e., giving preference to rule deactivation, correspond to consistent MCSs, and thus may be used for restoring consistency. However, the resulting options for repair may be too limiting for certain application domains. In our example, the option to disregard the allergy and prescribe strong antibiotics is missed. Rule deactivation either ignores the X-Ray, respectively blood test results, or that atypical pneumonia requires strong antibiotics, with the effect that either the disease is ignored at all, or a medication is suggested which might be to weak.

The study of more specific preference relations between diagnoses is a further research issue in our ongoing project on inconsistency management for MCSs. In addition to an investigation of abstract properties, preferences between diagnoses that emerge from application specific preferences on bridge rules or contexts, e.g., trust levels, are of particular interest. Moreover, we currently investigate approximations of diagnoses given incomplete information (when context knowledge is not fully disclosed), e.g., due to information hiding or security concerns.

Our aim is to provide support for resolving inconsistencies based on preferred diagnoses and inconsistency explanations. Note that due to presence of nonmonotonic contexts (witnessed by context $C_{4}$ in our example) the problem is more general than the problem of ontology merging (which may serve to build part of an MCS, however). Moreover, a decision for repair may need to take further domain knowledge into account, as illustrated by our example, where it is not obvious how to resolve the dilemma. Providing a declarative policy language for (semi-)automatic support for repair is the suggestive goal. Also, we plan to apply and evaluate the developed techniques in real world applications.

\section{References}

1. Brewka, G., Eiter, T.: Equilibria in heterogeneous nonmonotonic multi-context systems. In: AAAI. (2007) 385-390

2. Inoue, K., Sakama, C.: Abductive framework for nonmonotonic theory change. In: IJCAI. (1995) 204-210

3. Brain, M., Gebser, M., Pührer, J., Schaub, T., Tompits, H., Woltran, S.: Debugging ASP programs by means of ASP. In: LPNMR. (2007) 31-43

ISSN 1613-0073 (c) 2009 for the individual papers by the papers' authors. 


\title{
Relations as Context to Improve Multi-Target Tracking and Activity Recognition
}

\author{
Cristina Manfredotti ${ }^{12}$, Enza Messina $^{1}$, David Fleet ${ }^{2}$ \\ 1 DISCo, University of Milano-Bicocca, \\ \{manfredotti, messina\}@disco.unimib.it \\ 2 Computer Science Dept, University of Toronto, \\ \{cristina, fleet\}ecs.toronto.edu
}

\begin{abstract}
The explicit recognition of the relationships between interacting objects can improve the understanding of their dynamic model.

In this work, we investigate the use of Relational Dynamic Bayesian Networks to represent the dependencies between objects behavior in the context of multitarget tracking. We propose a new formulation of the transition model that accommodates for First-Order Logic relations and we extend the Particle Filter algorithm in order to directly track relations between targets.

Many applications can benefit from this work, as activities recognition, traffic monitoring, strategic analysis, sports coaching and others. We present some results about activity recognition in monitoring Canadian costal borders.
\end{abstract}

Key words: Multi Target tracking, Probabilistic Relational Models, Bayesian Filtering, Particle Filtering.

\section{Introduction and Motivations}

Context interpretation and context-based reasoning have been shown to be key factors for Computer Vision in the development of algorithms for object recognition [3]. In this domain the context is the scene where objects are located and the knowledge about it is expressed by the beliefs over the scene [4].

In this paper we deal with moving objects and we refer the concept of context to "what is happening around the object we are tracking". Knowing the scene can improve the objects recognition task and the knowledge about the identity of the objects improves the belief over the scene; knowing what is happening in the scene (which "relations" are believed to be true in the scene) can improve the tracking and the knowledge about the state of the objects can improve our knowledge about the relation between the objects in the scene (i.e. the context).

Consider, for example, the situation in which we have a group of people walking in a park. If we know they are walking together (i.e. if we have a certain belief over their relation), we know they will have a similar behavior or a similar motion. This will help us in tracking them. Moreover, taking into account the relations between objects can also allow us to recognize complex activities like, for example, the activity of "going to a pub together": single persons walking can be a simple fragment of a more complex activity that includes some people meeting, going in the same direction, waiting 
each other at different points and entering together into the pub. Dealing with relations between moving objects allows us to recognize a complex activity like this one from another similar one that can be the "catching the subway during rush hour": this complex activity also includes a group of people walking together in the same direction but those people will not wait for each other. In the last years Computer Vision has mostly dealt with the recognition of activities composed by the repetition of simple movements [12], instead those are examples of more complex activities that involve relations between objects and/or single actions during time.

In our work we model the context as a set of First-Order Logic relations using them in two principal ways:

- We will use relations to improve the efficiency of the tracking. The information contained in the relationships can improve prediction, resulting in a better estimation of objects trajectories.

- We will monitor relations as a goal in itself. This is the case in many applications like traffic prediction or consumer monitoring, anomaly detection or activity recognition.

In this work we consider Relational Dynamic Bayesian Networks (RDBNs) ${ }^{1}$, an extension of Probabilistic Relational Model [5] to dynamic domains, as a formalism to monitor relations between moving objects. In our RDBN-based model, relationships are considered as random variables whose values may change over time. While tracking the objects in the domain, we also track the evolution of their relationships. For this purpose, in the next sections we propose a formalization of a new dynamic model able to predict the future state of the objects taking into account their relations and we introduce a new version of Particle Filter (that we call Relational Particle Filter) that adapts to these settings. After presenting some preliminary results obtained on the Intelligent System Challenge 2008-2009 data set ${ }^{2}$, and a brief review of the literature, we conclude with some final remarks.

\section{Modeling and Inference}

A relational domain is a set of objects with relations between them. We will call the state $s$ of a relational domain relational state, and we define it as the set of instantiations of all the objects and their relations in the domain. Therefore, we can divide the relational state in two parts: the state of the objects ${ }^{3} s^{o}$ and the state of the relations $s^{r}$ and we will write: $s=\left[s^{o}, s^{r}\right]$.

A Relational Bayesian Network (RBN) is a directed acyclic graph whose nodes are First-Order Logic attributes or relations between objects in the relational domain and whose structure represents the causality between the nodes.

\footnotetext{
${ }^{1}$ The authors are aware of the works of Sanghai, Weld and Domingos on RDBNs; however the paper presenting their work has been retracted. Refer to: http://www.aaai.org/Library/JAIR/Vol24/jair24-019.php

${ }^{2} \mathrm{http}: / / \mathrm{www}$.intelligent-systems-challenge.ca/home/index.html

${ }^{3}$ We will use the terms state of objects and state of instantiations interchangeably.
} 
When we deal with dynamic, relational states evolve with time and RBNs has to be extended to RDBNs. A Relational Dynamic Bayesian Network is structured as a pair of RBNs $\left(B_{0}, B_{\rightarrow}\right)$, where $B_{0}$ represents the probability distribution over the state of the relational domain at time 0 and $B \rightarrow$ is a RBN of nodes at time $t$ whose parent are predicates at time $t-1$ or predicates at time $t$ and nodes at time $t-1$ without their parents.

In order to make inference in a multi-target setting, we need to extend the algorithms traditionally used in tracking to represent relations. As in classic tracking, the aim is to estimate the current posterior distribution of the state space $s_{t}$ conditioned to the sequence of observations $z_{1: t}$ up to time $t: p\left(s_{t} \mid z_{1: t}\right)$. This distribution is often called the target's belief (bel $\left.\left(s_{t}\right)\right)$.

The tracker predicts the probability distribution of the future state $s_{t}$, given the knowledge about the current state $s_{t-1}$, by means of a state transition model $p\left(s_{t} \mid s_{t-1}\right)$. Once measurements about the state at time $t\left(z_{t}\right)$ are acquired, the state is filtered using the sensor model $p\left(z_{t} \mid s_{t}\right)$ that relates (potentially noisy) measurements to the state.

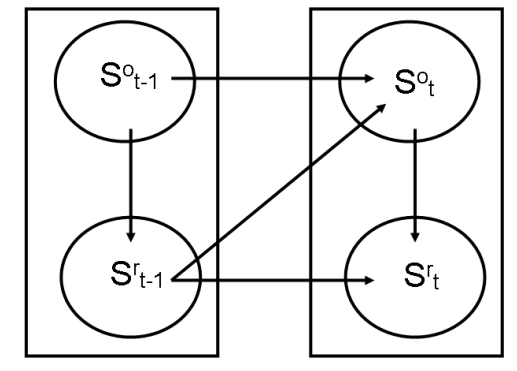

Fig. 1. Relational Transition Model. Arrows indicate probabilistic dependence between variables.

To extend the traditional tracking algorithms to represent relations we introduce the following components:

The relational transitional model $p\left(s_{t} \mid s_{t-1}\right)=p\left(s_{t}^{o}, s_{t}^{r} \mid s_{t-1}^{o}, s_{t-1}^{r}\right)$ is a joint probability of the state of all instances and relations. We assume that the state of relations is not directly affected by the state of the objects at the previous time step (see Figure 1). Therefore the transition model can be rewritten as:

$$
p\left(s_{t}^{o}, s_{t}^{r} \mid s_{t-1}^{o}, s_{t-1}^{r}\right)=p\left(s_{t}^{o} \mid s_{t-1}^{o}, s_{t-1}^{r}\right) p\left(s_{t}^{r} \mid s_{t-1}^{r}, s_{t}^{o}\right) .
$$

The sensor model $p\left(z_{t} \mid s_{t}\right)$ gives the probability of the state at time $t$ given the measurements obtained at the same time. We assume the relations to be not directly measurable, so the observation $z_{t}$ is independent of the relations between objects:

$$
p\left(z_{t} \mid s_{t}\right)=p\left(z_{t} \mid s_{t}^{o}, s_{t}^{r}\right)=p\left(z_{t} \mid s_{t}^{o}\right) .
$$

Under the Markov assumption and the conditional independence of the data given the state, we can use a Bayesian filter algorithm to compute the belief of the relational 
state:

$$
\operatorname{bel}\left(s_{t}\right)=\alpha p\left(z_{t} \mid s_{t}^{o}\right) \widetilde{\operatorname{bel}}\left(s_{t}\right)
$$

where $\alpha$ is a normalization constant and $\widetilde{b e l}\left(s_{t}\right)$ is the prediction done over the system $\left(p\left(s_{t}^{o}, s_{t}^{r} \mid z_{1: t-1}\right)\right)$ that can be computed as:

$$
\widetilde{\operatorname{bel}}\left(s_{t}\right)=\int p\left(s_{t}^{o}, s_{t}^{r} \mid s_{t-1}^{o}, s_{t-1}^{r}\right) \operatorname{bel}\left(s_{t-1}\right) d s_{t-1} .
$$

According to the state transition model (Equation 1), we can write Equation (4) as:

$$
\widetilde{\operatorname{bel}}\left(s_{t}\right)=\int p\left(s_{t}^{o} \mid s_{t-1}^{o}, s_{t-1}^{r}\right) p\left(s_{t}^{r} \mid s_{t-1}^{r}, s_{t}^{o}\right) \operatorname{bel}\left(s_{t-1}\right) d s_{t-1} .
$$

In the most general case we can represent the two partial transition models of Equation (1) by a First Order Logic Tree (FOPT) ${ }^{4}$. We will introduce an example of FOPT when dealing with the experiments.

\subsection{Relational Particle Filter}

The specific and complex probabilistic nature of the presented setting makes impossible to use filters that require a probabilistic function in closed form, such as the Kalman filter. To solve this issue we developed an extension of the Particle Filter (PF) algorithm whose properties are appealing for our case.

The PF algorithm [1] is a Monte Carlo method that approximates the required posterior density function by a set of random samples with associated weights and computes estimates based on these samples and weights. As the number of samples becomes very large, the Monte Carlo approximation to the correct posterior improves and the PF approaches the optimal Bayesian estimate.

We integrate the relational transitional model introduced in Equation (1) in a new Relational Particle Filter (RPF), shown in Algorithm (1).

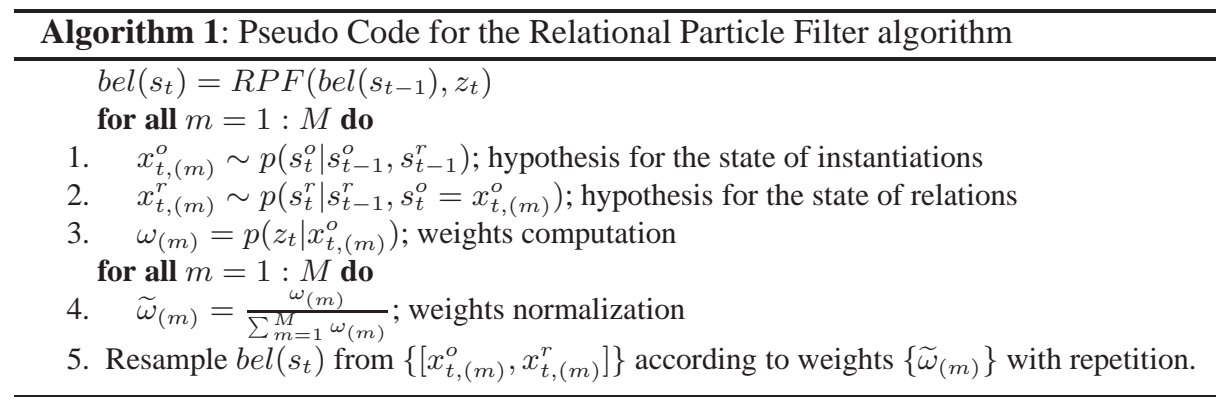

\footnotetext{
${ }^{4}$ A FOPT (also known as First Order Decision Diagram [2]) is Probabilistic Tree whose nodes are First Order Logic formulas.
}

ISSN 1613-0073 @ 2009 for the individual papers by the papers' authors. 
A particle $\left(x_{t,(m)}\right)$ is a representation of the state. For this reason, in our setting, it is divided in two parts: the part of the objects $x_{t,(m)}^{o}$ and the part of the relations $x_{t,(m)}^{r}$. (see Figure 2(a)). The part of the particle relative to the instantiations is sampled according to $p\left(s_{t}^{o} \mid s_{t-1}^{o}, s_{t-1}^{r}\right)$ (Line 1), subsequently the part of the particle relative to the relations is sampled according to the second part of the relational transition model (Line 2). When the measurement is acquired, particles are weighted according to the sensor model (Line 3). The sensor model takes into account only the part of the particles relative to the objects, since the particles are composed by two parts, also the parts associated to the relations are weighted. After the weighting step, weights are normalized (Line 4) and the set of particles for the next iteration is extracted according to the normalized weights in the resampling step (Line 5).

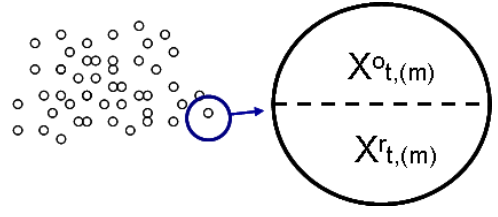

(a) Particle representation.

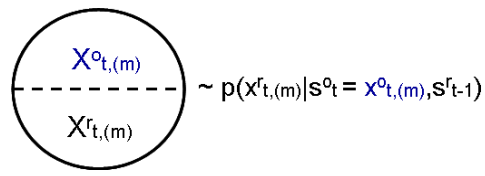

(c) Second step of hypothesis.

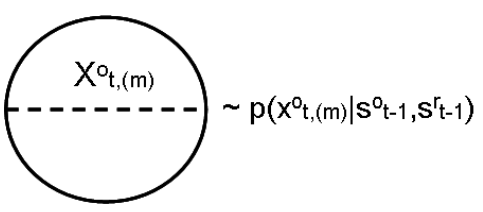

(b) First step of hypothesis.

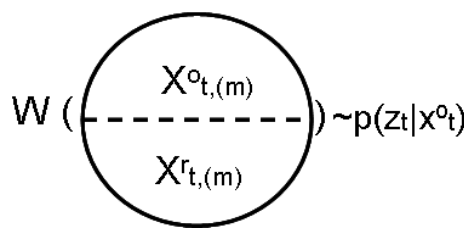

(d) Particle weighting.

Fig. 2. Cartoon representation of the proposed algorithm.

\section{Experiments}

We evaluated the proposed method on a synthetic data set that is based on the Intelligence System Challenge 2008-2009 data set. We chose to use this data set because it is easier than a real data set but still challenging. The data set contains the description of the events happened in the sea; each element of the data set reports the tracks of two boats participating in an event (i.e. Rendezvous, PickUp and Avoidance) together. At each time interval at most one event takes place.

We are particularly interested in the case where there is uncertainty about the participants taking part in an event, in order to demonstrate the advantage of maintaining 
beliefs over the set of possible relations. In order to test our relational particle filter algorithm for activity recognition in a more challenging scenario with multiple targets, we use the original data set to build a new synthetic data set of 120 situations (either rendezvous or avoidance), obtained by pairing two encounters randomly sampled from the original data set. In this way, four ships are present at the same time in the scene.

In the experiment, we consider the task of detecting a rendezvous between a yacht and a fisher ship. After describing the setting of our experiments, in the next subsection, we report some results.

\subsection{Settings and Results}
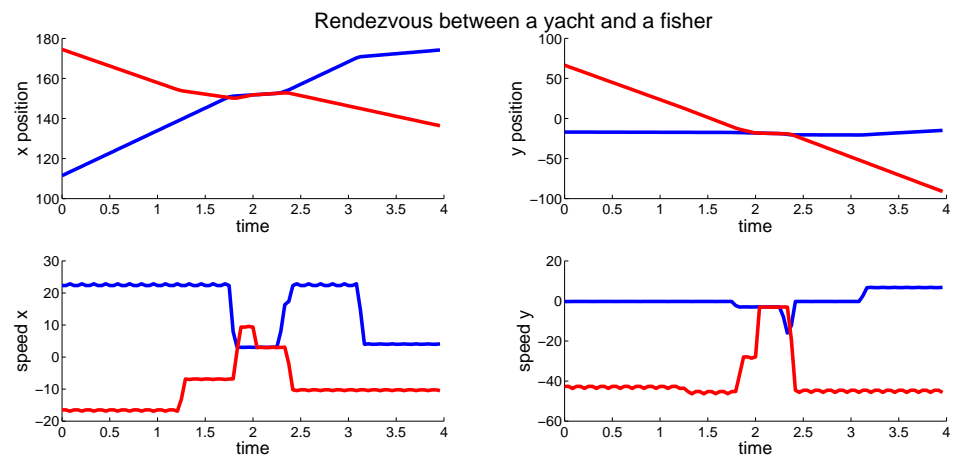

Fig. 3. Example of Rendezvous.
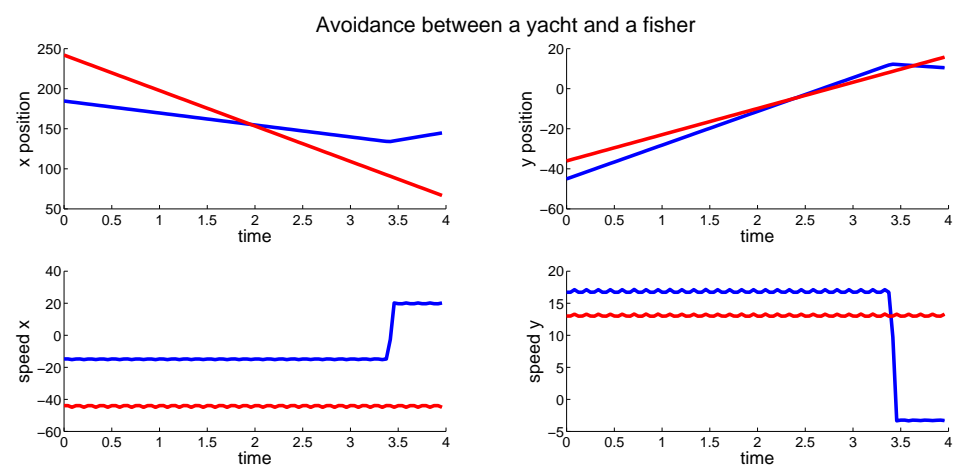

Fig. 4. Example of no relation. 
We used the data set to estimate the prior for the event Rendezvous between a Fisher and a Yacht (33/80). Then, we examined the data relative to the encounters in order to acquire information about the two different events (rendezvous or avoidance) that can be used to predict the relation. In particular, we focused on the variation of speed of the two targets. Consider for example, the rendezvous in Figure (3): the two ships come closer and both progressively reduce their speed until a nearly-zero value. Different is the case of ships that are avoiding each other (thus not in relation according to our model), one maintains its speed and the other decelerates (Figure (4)).

From the images it is also possible to notice the three-phases which characterize the event of rendezvous: ships approach each other reducing their speed in the first phase, they travel in the same direction with nearly-zero speed in the second phase and finally they go apart and at least one of them change its speed. Our relational transition model takes into account these three different phases allowing to detect when the event starts and when it finishes but also allowing to understand if two ships can be in relation (since a ship can be in relation only with another ship).

An example of the relational transition model used in our experiments is given in Figure (5) and in Figure (6) .

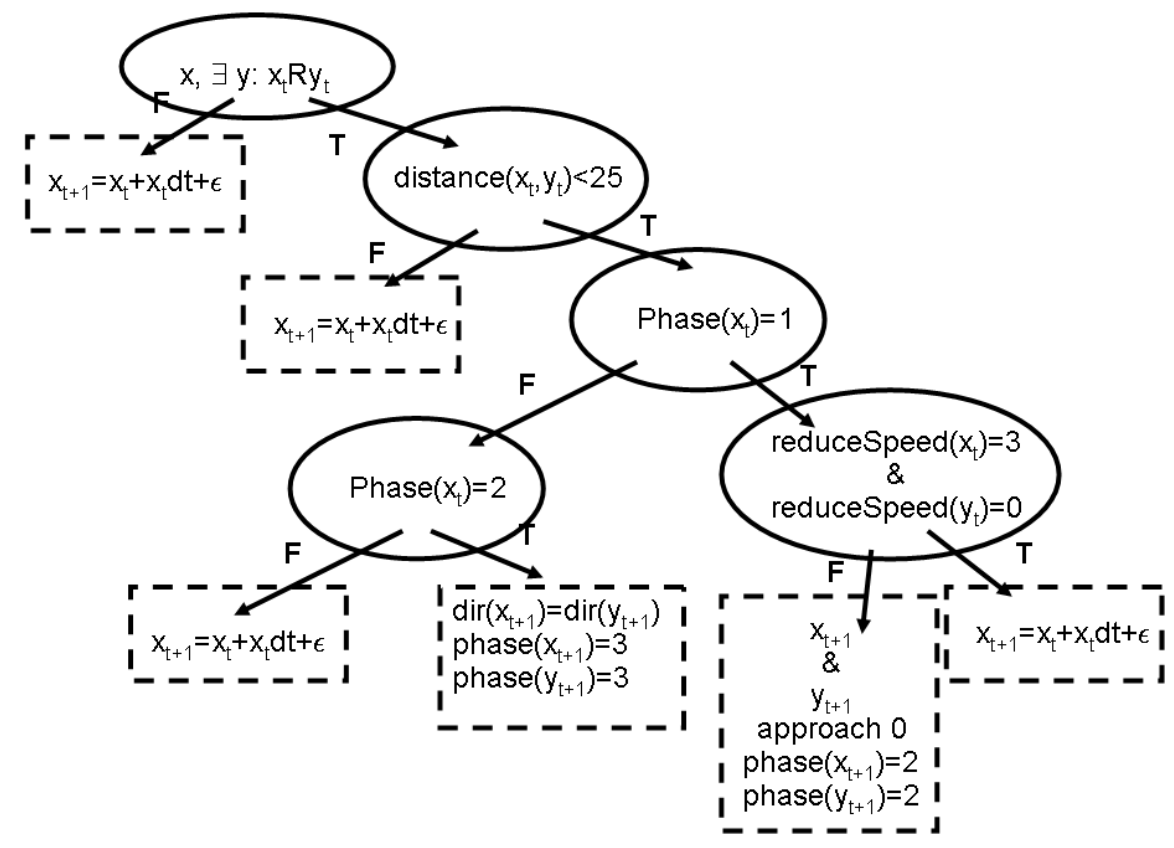

Fig. 5. FOPT for $p\left(s_{t}^{o} \mid s_{t-1}^{o}, s_{t-1}^{r}\right)$.

We ran the experiments on each of the 120 sets of four tracks in the data set. In table (1) we show the accuracy of our method for the rendezvous detection compared 


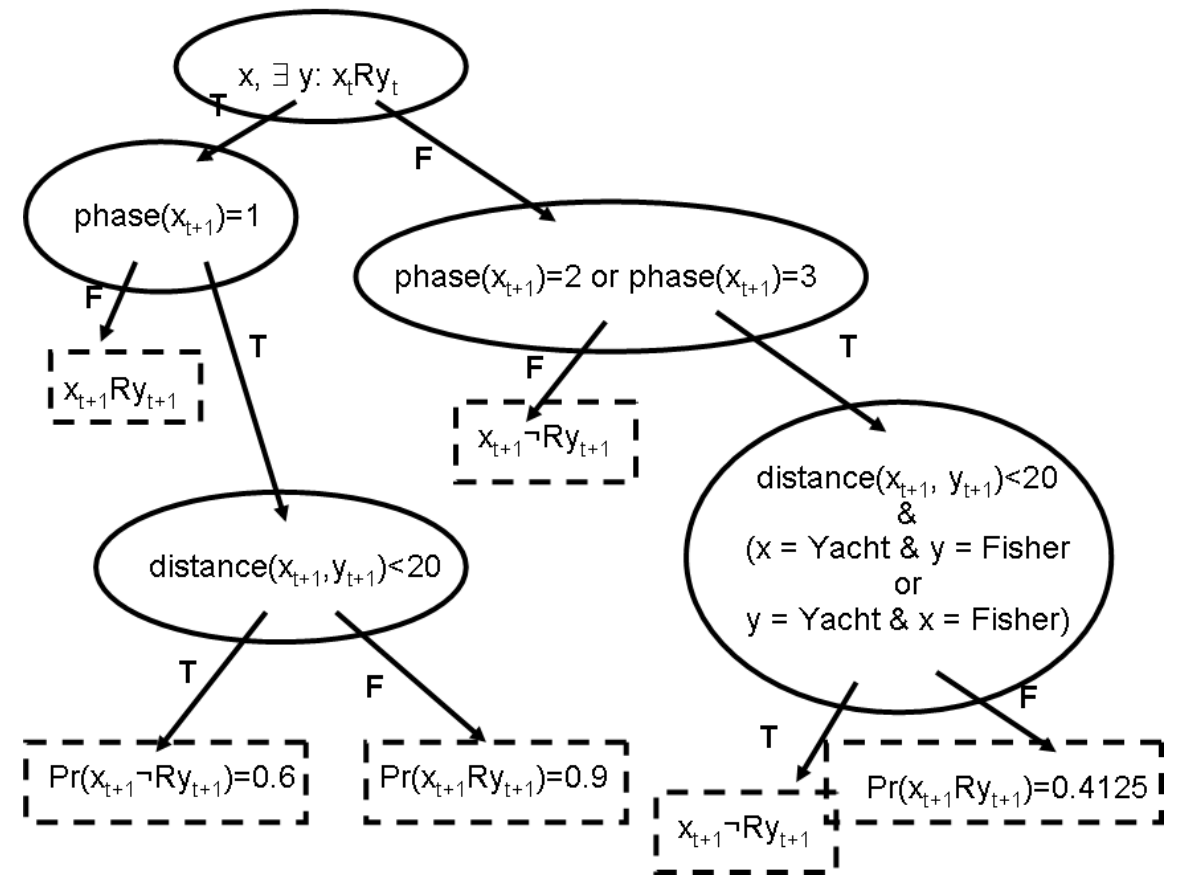

Fig. 6. FOPT for $p\left(s_{t}^{r} \mid s_{t-1}^{r}, s_{t}^{o}\right)$.

to the accuracy of a method that randomly choses which boats are in relation. In the table it is also reported the average tracking error of the RPF algorithm compared to a $\mathrm{PF}$ algorithm that does not take into account relations. The tracking error is computed as the distance between the trajectories acquired by the particle filter (at each time-step, it averages the position considering the states of all particles) and the real trajectories.

\begin{tabular}{|c|cc|c|}
\hline method & TP ration TN ratio & Tracking Error $(\mathrm{km}$, mean $)$ \\
\hline RPF & 0.4545 & 0.7235 & 1.8379 \\
\hline $\begin{array}{c}\text { PF } \\
\text { random choice }\end{array}$ & 0.4444 & 0.4841 & 3.3906 \\
\hline
\end{tabular}

Table 1. In columns TP ratio and TN ratio the ratio over the 120 set of tracks of true positive and true negative is reported for our method and a random choice method. In the last column the average tracking error for our method (RPF) and a method that does not take into account relations $(\mathrm{PF})$ is reported. 


\subsection{Related Works}

Our work is at the intersection of work in Probabilistic Relational Models, that to our knowledge have never before considered applications in tracking, and Computer Vision, where often heuristics are used to improve tracking, but not with a systematic account of relationships between targets.

Recently there has been increasing interest in models that extend probabilistic reasoning to First Order Logic to exploit redundancies observed in the worlds ([5], [6]). In this setting, many relational inference algorithms proceed by first fully instantiating the First-Order relations and then working at the propositional level. In [10] an inference algorithm that instantiates relations only as needed is presented, but this algorithm can deal only with static domains as the relations are not supposed to change over time. Moreover, our model is different from the one presented in [9], where the concept of class is used to develop an inference system able to deal with a large number of heterogenous objects. We use First-Order Logic to explicitly represent relationships between objects to improve the inference task. Our method is potentially applicable to situations with a large number of objects as well.

Hybrid states models have been used to deal with complex tracking tasks [7]. They combined continuous-valued dynamic with a discrete state of the world (context) encoding which switching dynamic is performed jointly with tracking. Our system uses relations as representations of the context of each object instead of the context of the entire world. The explicit recognition of the relations of each object allows us to deal with much more complex tracking tasks. Moreover, the use of First Order Logic (as opposed to predicative logic) generalizes our models to different domains.

In [8] the recognition of complex activity (temporally extended activities that can be fragmented in simple ones) is based on context-free grammar. They decouple the recognition task in two levels: a lower level that detects single simple activities that are the inputs for the stochastic context-free grammar used as a "bag of words" for a parsing mechanism. Instead, our approach does not decouple the recognition task, but seek to take advantage from the tracking, that provides the detection of simple activities, to recognize the temporally extended activity and from the knowledge about the complex activity to improve the tracking.

In [11], the authors address the problem of activity recognition using First Order Logic rules and Markov Logic Networks to represent common sense domain knowledge. Differently from the method we are proposing, the inference task is performed off-line: they perform probabilistic inference for input queries about events of interest already happened. We seek, instead, to perform an on-line probabilistic inference of both the state of the domain and the activities.

\section{Conclusions}

In this paper we presented a technique based on relational Bayesian reasoning in order to address the problem of activity recognition and tracking. We presented an extension of particle filter, called relational particle filter, that can be used to make inference. From our preliminary results we can conclude that our method can help to identify the type of 
encounter that the targets are engaging. Moreover we have shown how using relations as context can improve the tracking task. Compared to hybrid state model techniques, we are able to model the problem with a single dynamic model and the state representation is much more compact.

There are a number of possible applications of this approach in problems where there is the need of monitoring a situation from sensed data (video surveillance, homelandsecurity, etc.) that we are interested to consider for future works.

\section{References}

1. S. Arulampalam, S. Maskell, and N. Gordon. A tutorial on particle filters for online nonlinear/non-gaussian bayesian tracking. IEEE Transactions on Signal Processing, 50:174188, 2002.

2. S. J. C. Wang and R. Khardon. First order decision diagrams for relational mdps. JAIR, 31:431-472, 2008.

3. A. A. E. Derek Hoiem and M. Hebert. Putting objects in perspective. In Proc. IEEE Computer Vision and Pattern Recognition (CVPR), volume 2, pages 2137 - 2144, June 2006.

4. G. Elidan, G. Heitz, and D. Koller. Learning object shape: From drawings to images. In Proceedings of the Conference on Computer Vision and Pattern Recognition (CVPR), 2006.

5. N. Friedman, L. Getoor, D. Koller, and A. Pfeffer. Learning probabilistic relational models. In IJCAI, pages 1300-1309, 1999.

6. L. Getoor, N. Friedman, D. Koller, and B. Taskar. Learning probabilistic models of link structure. Journal of Machine Learning Research, 3:679-707, 2002.

7. M. Isard and A. Blake. A mixed-state condensation tracker with automatic model-switching. In ICCV, pages 107-112, 1998.

8. Y. A. Ivanov and A. F. Bobick. Recognition of visual activities and interactions by stochastic parsing. IEEE Trans. Pattern Anal. Mach. Intell., 22(8):852-872, 2000.

9. B. Milch and S. J. Russell. General-purpose mcmc inference over relational structures. In UAI, 2006.

10. H. Poon, P. Domingos, and M. Sumner. A general method for reducing the complexity of relational inference and its application to mcmc. In AAAI, pages 1075-1080, 2008.

11. S. D. Tran and L. S. Davis. Event modeling and recognition using markov logic networks. In $E C C V$ (2), pages 610-623, 2008.

12. R. S. Yan Ke and M. Hebert. Event detection in crowded videos. In IEEE International Conference on Computer Vision, October 2007. 


\title{
Who, Where, What, and When: Towards Characterizing Contexts in Pervasive Environments
}

\author{
Alessandra Mileo \\ Department of Informatics, Systems and Communication, University of \\ Milan-Bicocca, viale Sarca 336/14, I-20126 Milan \\ alessandra.mileo@nomadis .unimib.it
}

Introduction. A key challenge in pervasive systems is that of identifying and developing appropriate formal approaches for understanding and reasoning about contexts. This is a difficult task since there is no common agreement on what constitutes a context and what its properties are. Although the literature contains a rich variety of context models $[1,2]$, most of the resulting implementations are domain-dependent and lack powerful reasoning capacities. Preliminary work on logic-based reasoning about contexts has been proposed in [3, 4], using bridge rules and argumentation theory. However, application-wise there are still open questions on how to model context-related pieces of knowledge in general domains, so that appropriate reasoning techniques can be applied. In order to put the basis for a comprehensive theory of contexts one should take into account the general structure of knowledge needed to represent contexts, in particular, what is intended as a context in pervasive environments and which components/properties should be identified and described. System properties that are of interest for the specification of contexts should be also considered. Motivated by application challenges, this short paper highlights observations that can be used to address these issues.

Generalization of Context in Pervasive Environments. Previous investigations of context models in pervasive environments revealed that within contexts certain entities are more important than others in practice. These are location, identity, activity and time $[5,6]$. In pervasive environments, the more intuitively relevant aspects of the context in which a person acts are who she is, which resources/entities are related to her, where she is and when. The representation of knowledge about contexts is very similar to the structure of a sentence in natural language, where actors are the subjects and the objects, verbs are relations and sensor data are the adjectives in that they assign a value to attributes of the actors. As an example, in a home monitoring scenario, a generalization of actors may correspond to the following entities: Person to be monitored, Room and Area for localization and Object for resources. If attributes correspond to sensor data, relations that we consider interesting depend on the task we want to solve (localization of a person, reaction from the system, identification of an action, etc.). The fact that a relation holds or not should be the result of reasoning (commonsense, default, temporal) on the knowledge we want to characterize. 
To enhance generality and modularity of the representation, we suggest to represent entities at a primary level while all the other pieces of sensed information are at a second level and can be indexed as attributes of the entities at primary level. As for the relations between subjects, objects, and places in pervasive environments the basic interesting relations that should be formalized are spatial (being close/far, being in/out), temporal (occurring before/after) and causal (occurring because of something).

Properties. In a given context, attributes and relations need to be associated to a time stamp. In this way, the reasoning system can take into account their dynamic evolution to characterize aspects such as commonsense, temporal reasoning, belief revision and update. In general the temporal evolution of a context is one of the most important aspect to be correctly and formally characterized. Additional properties may be needed to determine what is true in a given context where certain objects, attributes and entities have been defined. These properties should make it possible to express belief merging and belief revision/update in contexts.

Conclusions. We believe reasoning about contexts became harder and harder because of the proliferation of very personal representations of context knowledge in terms of admissible relations, their arity and their properties, which have been developed ad-hoc for very diverse specific applications. Our attempt is that of providing a more general and modular representation of context knowledge. The intuition is that in this way multi-context reasoning could be supported by the verification of formal properties at a knowledge representation level, in the same way as human deduction is based on roles of the grammatical components of a sentence.

\section{References}

1. Baldauf, M., Dustdar, S., Rosenberg, F.: A survey on context-aware systems. International Journal of Ad Hoc and Ubiquitous Computing (2007) 263-277

2. Strang, T., Linnhoff-Popien, C.: A context modeling survey. In: Proc. of the Workshop on Advanced Context Modelling, Reasoning and Management. (2004)

3. Brewka, G., Eiter, T.: Equilibria in heterogeneous nonmonotonic multi-context systems. In: Proc. of the 22nd AAAI Conference on Artificial Intelligence, July 22-26, 2007, Vancouver, British Columbia, Canada, AAAI Press (2007) 385-390

4. Brewka, G., Eiter, T.: Argumentation context systems: A framework for abstract group argumentation. In: Proc. of LPNMR09, Springer-Verlag, In press (2007)

5. Ryan, N.S., Pascoe, J., Morse, D.R.: Enhanced reality fieldwork: the context-aware archaeological assistant. In Gaffney, V., van Leusen, M., Exxon, S., eds.: Computer Applications in Archaeology 1997. British Archaeological Reports, Tempus Reparatum (1998)

6. Schilit, B., Adams, N., Want, R.: Context-aware computing applications. In: Proc. of the Workshop on Mobile Computing Systems and Applications, IEEE Computer Society (1994) 85-90 


\title{
A Constructive Modal Semantics for Contextual Verification
}

\author{
Giuseppe Primiero* \\ Centre for Logic and Philosophy of Science \\ University of Ghent (Belgium) \\ Blandijnberg 2, room 231, 9000 Gent \\ Giuseppe.Primiero@UGent.be
}

\begin{abstract}
This paper introduces a non-standard semantics for a modal version of constructive $K T$ for contextual (assumptions-based) verification. The modal fragment expresses verifiability under extensions of contexts, enjoying adapted validity and (weak) monotonocity properties depending on satisfaction of the contextual data.
\end{abstract}

\section{Background and Motivation}

Modelling contexts is a crucial issue for knowledge representation and problem solving tasks ([6]). The constructive treatment of contexts, interpreted as meaning determining environments in a pragmatic setting for indexical expressions ([5]) or as databases for information retrieval, is characterized by the reduction of assumptions to verified instances. From a logical viewpoint, the formulation of a constructive contextual possible worlds semantics is an interesting challenge to pair the syntactic calculi presented in [2] for staged computation, in [9] for an operational semantics that quantifies over contexts and in [10] for a constructive type theory with refutable assumptions.

Our constructive contextual semantics presents two novel aspects: the representation of verification processes under open (non reduced) assumptions, and their modelling in a contextual dynamics. These properties are given by interpreting necessity as verifiability in the empty context of assumptions preserved to all extensions, and possibility as restricted validity. When performing queries on ontologies, one wants the theory to deal with validity of varying contextual values:

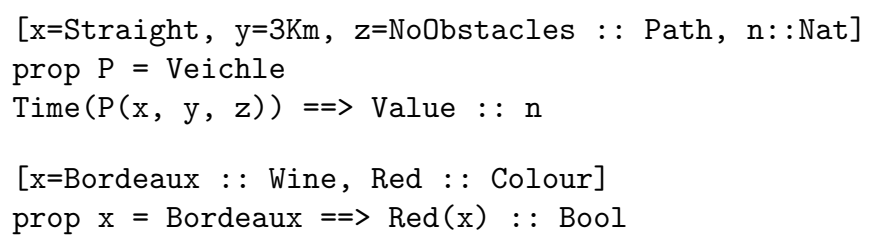

* Postdoctoral Fellow of the Research Foundation - Flanders (FWO). Affiliated Senior Researcher IEG, Oxford and GPI, Hertfordshire (UK). 
This dynamics should be admitted both at the typing level (e.g. with type declaration City in place of Wine, resulting in a different output) and at the value level (e.g. with type value $30 \mathrm{~km}$ in place of $3 \mathrm{~km}$, resulting in a different computation $z$ ). The main applications are knowledge processes with unverified information, programming under contextual verification and output correcteness in distributed and staged computation.

\section{Knowledge with Local and Global Contexts}

The language $\mathcal{L}_{\text {int }}$ is the union of two fragments $\mathcal{L}_{\text {int }}=\left\{\mathcal{L}^{\text {ver }}, \mathcal{L}^{\text {inf }}\right\} . \mathcal{L}^{\text {ver }}$ is a positive (intuitionistic) language for direct verification processes, built in a standard way from propositional variables $\mathcal{P}=\{A, B, C, \ldots\}$, the propositional constant $T$, propositional unary and binary connectives $\neg, \&, \vee, \supset$. $\mathcal{L}^{\text {inf }}$ is an extension of the previous language with $\perp$ and modal operators $\square, \diamond$, obtained by defining the satisfaction relation in a context $\Gamma$. A set of knowledge states $\mathbb{K}=\left\{K_{i} \mid i \in \mathbb{I}\right\}$ is a finitely enumerable collection of finite sets of evaluated (modal and non-modal) formulas from $\mathcal{L}_{\text {int }}$; each state is decorated with indices $\mathbb{I}=\{i, j, k, \ldots\} ; \mathcal{V}=\left\{x_{1}, x_{2}, \ldots\right\}$ denotes a finite set of free variables.

A model $M^{\text {ver }}=\{\mathbb{K}, \leq, R, v\}$ is normal model with an accessibility relation on ordered states $K_{i} \leq K_{i} \in \mathbb{K}$ on which monotonicity is preserved for valuating propositional letters by a standard function $v$. Contexts $\Gamma, \Gamma^{\prime}$ are sets of valuation functions from $\mathcal{V}$ to contents in a knowledge state. The partial order $\leq^{\gamma}$ holds for knowledge states on the basis of the relevant informational contexts, where the function $\gamma$ defines the extension of $\Gamma$ holding for a given $K_{i}$ to $\Gamma^{\prime}$ of $K_{j}$ $\left(K_{i} \leq^{\gamma} K_{j}\right)$, with at least one new propositional content assumed in $K_{j \geq i}$. Each value obtained in context can be seen as the parametric module of the new language, collected into a strucutred list. $v_{M^{i n f}}, K_{i} \vDash \Gamma A$ is read as: " $A$ is verified in $K_{i}$ on the basis of information $\Gamma$ " and is based on the function $\gamma:=\mathcal{V} \mapsto \mathbb{K}$, such that $\gamma$ verifies $A \in K_{j}$ iff $M^{\text {ver }}\left(K_{i \mid i \leq j \in \mathbb{I}}\right) \not \models \neg A$. A model $M^{\text {inf }}=\left\{\mathbb{K}, \leq^{\gamma}, R, v\right\}$, has $R$ as a symmetric accessibility relation on $\mathbb{K}$ induced by $\leq \gamma$ and $v$.

An informational context is the dynamic structure of information which specifies the actual program (or theory) against which the knowledge state is valid. The additional two specific clauses for modalities in this language interpret contextual dynamics:

- $v_{M}, K_{i} \vDash^{\Gamma} \square A$ iff for any function $\gamma$ it holds $K_{i} \vDash^{\Gamma \leq \gamma} A$;

$-v_{M}, K_{i} \vDash^{\Gamma} \diamond A$ iff there is a function $\gamma$ for which it holds $K_{i} \models^{\Gamma \leq \gamma} A$.

Monotonicity for $\mathcal{L}^{i n f}$ is expressed under contextual constraints:

Lemma 1 (Contextual Monotonicity for $\mathcal{L}^{\text {inf }}$ ). If $K_{i} \vDash^{\Gamma} \top$ and for all $\gamma$, $\Gamma \leq^{\gamma} \Gamma^{\prime} \vDash \top$, then $K_{j} \models^{\Gamma^{\prime}} \top$ and if $K_{i} \models{ }^{\Gamma} A$ then $K_{j} \models{ }^{\prime} A$.

Introducing the distinction between global and local assumptions (see [4]) allows to reduce derivability and consequence relations of the two procols to a unified frame. 
Definition 1 (Global Context). For any context $\Gamma$, the global context $\square \Gamma$ is given by $\bigcup\left\{\square A_{1}, \ldots, \square A_{n}\right\}$ such that $\gamma:=x \mapsto A_{i} \in \Gamma$.

Definition 2 (Local Context). For any context $\Gamma$, the local context $\diamond \Gamma$ is given by $\bigcup\left\{\circ A_{1}, \ldots, \circ A_{n} \mid \circ=\{\square, \diamond\}\right\}$ and $\gamma:=x \mapsto A_{i} \in \Gamma$ and $\exists A_{i}$ such that $\diamond A_{i}$.

The resulting system has a correspondingly formulated consequence relation: $K_{i} \vDash^{\square \Gamma} A$ iff for every $\gamma$, it holds $K_{i} \vDash^{\Gamma \leq \gamma} A ; K_{i} \vDash^{\diamond \Gamma} A$ iff for some $\gamma$ it holds $K_{i} \models^{\Gamma \leq \gamma} A$. The class of models $\mathcal{M}^{\left(\mathcal{L}^{\text {ver }} \cup \mathcal{L}^{i n f}\right)}$ is equivalent to that of contextual $K T$ (see [1], [3], [7]) with $\square$ and $\diamond$ :

Theorem 1. The system $C K T_{\square, \diamond}$ is sound and complete with respect to the union class $\mathcal{M}^{\left(\mathcal{L}^{\text {ver }} \cup \mathcal{L}^{\text {inf }}\right)}$; i.e. for every set of formulae $\Gamma$ and formula $A$, it holds $\Gamma \vdash_{C K T_{\square, \diamond}} A$ iff either $\vDash \wedge \Gamma \supset A$, or $\vDash \square \Gamma A$, or $\vDash \diamond \Gamma A$.

\section{References}

1. Alechina, N. Mendler, M., de Paiva, V., Ritter, E., "Categorical and Kripke Semantics for Constructive S4 Modal Logic", Proceedings 15th Int. Workshop on Computer Science Logic, CSL'01, Paris, France, 10-13 Sept. 2001, pp. 292-307, Springer-Verlag, 2001.

2. Davies, R., Pfenning, F., "A modal Analysis of Staged Computation", Journal of the ACM, vol.48, n.3, pp.555-604.

3. de Paiva, V., "Natural deduction and context as (constructive) modality", in Proceedings of the $4^{\text {th }}$ International and Interdisciplinary Conference - CONTEXT03, Stanford, vol. 2680 of Lecture Notes in Artificial Intelligence, Springer Verlag, 2003.

4. Fitting, M., Basic Modal Logic, in D.M. Gabbay, C.J. Hogger, J.A. Robinson (eds), Handbook of Logic in Artificial Intelligence and Logic Programming, vol. 4, 368449, Oxford University Press, 1994.

5. Giunchiglia, F., Bouquet, F., "Introduction to contextual reasoning. An Artificial Intelligence perspective", in: B. Kokinov (ed.), Perspectives on Cognitive Science, vol. 3, NBU Press, Sofia, 1997, pp. 138-159.

6. McCarthy, J., "Notes on formalizing context", in Proceedings of the $13^{\text {th }}$ Joint Conference on Artificial Intelligence (IJCAI-93), 1993.

7. Mendler, M., de Paiva, V., "Constructive CK for Contexts", in Proceedings of the first Workshop on Context Representation and Reasoning - CONTEXT05, Stanford, 2005.

8. Nanevski, A., Pfenning, F., Pientka, B., "Contextual Modal Type Theory", in ACM Transactions on Computational Logic, vol. 9(3), pp.1-48, 2008.

9. Pientka, B., Dunfield, J., "Programming with Proofs and Explicit Contexts", Proceedings of the 10th international ACM SIGPLAN Conference on Principles and Practice of Declarative Programming, Valencia, SPain, ACM, pp.163-173.

10. Primiero, G., "Constructive contextual modal judgments for reasoning from open assumptions", Technical Report 542, Centre for Logic and Philosophy of Science, Ghent University, 2009; http://logica.ugent.be/centrum/preprints/primiero_open_assumptions.pdf. 